\title{
Predicting Logarithm of Real Numbers to any Real Number Base
}

\author{
Mohammad Asfaque ${ }^{1}$, Jeevan Kafle ${ }^{2, *}$ \\ ${ }^{1}$ St. Xavier's College, Maitighar, Kathmandu, Nepal \\ ${ }^{2}$ Central Department of Mathematics, Tribhuvan University, Kirtipur, Nepal \\ *Corresponding Author: jeevan.kafle@cdmath.tu.edu.np
}

\begin{abstract}
This paper aims to propose an algorithm that can predict the values of logarithmic function for any domain and for any bases with minimum possible error using basic mathematical operations. It talks about a univariate quadratic function that overlaps with the graph of the common logarithmic function to some extent, and using this resemblance to fullest advantage. It proposes a formula and certain algorithms based on the same formula.
\end{abstract}

Keywords: Logarithm, Quadratic Function, Coincidence, Algorithm, Error

\section{Introduction}

The logarithmic function is the inverse function to exponential function. That means the logarithm of a given number $x$ is the exponent to which the base $n$ must be raised to get the given number $x[1,2]$. Let $x=n^{y}$ be an exponential function then, $y$ $=\log _{n} x$, where $n>0(n \neq 1)$ is the logarithmic function with base $n$. The domain of the logarithmic function includes all positive real numbers and the range includes all real numbers $[1,2]$. Logarithm to base 10 is called common logarithm which is simply written as $\log x$, [3] whereas $\operatorname{logarithm}$ to the base $e$ is called natural logarithm, and it is written as $\ln x[4]$.

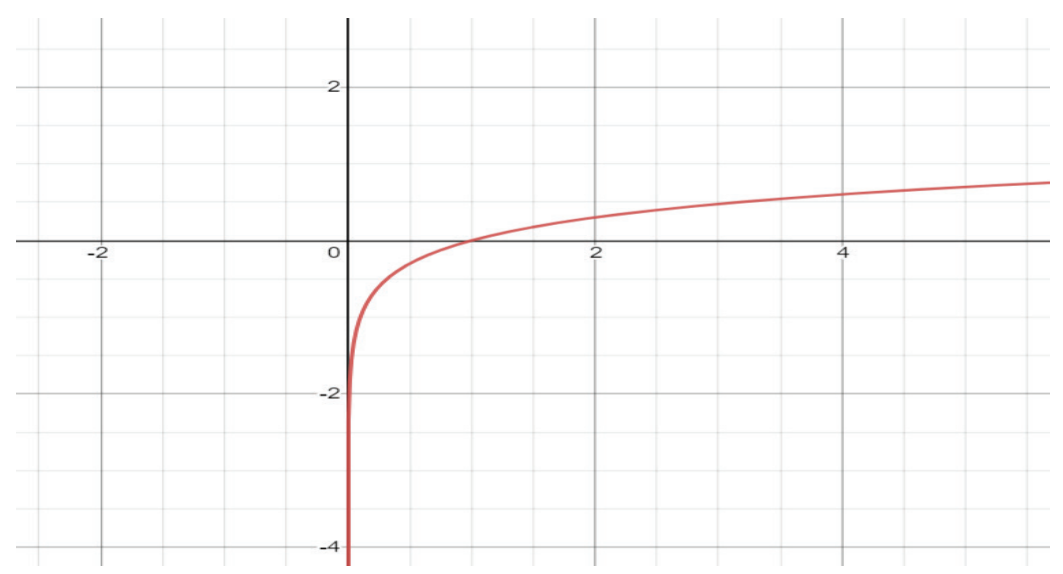

Figure 1: Graph of $y=\log x$ 


\section{Resemblance of a Quadratic Function with the Common Logarithmic Function}

A quadratic function that is $f(x)=0.19 x-0.01 x^{2}$ passes close to the common logarithmic function $y=\log x$ (see in Fig. 2). These two functions are completely different by their nature, but their overlapping for quite a range makes things easy for us. They start overlapping at somewhere between $x=2.5$ and $x=3$ (see in Fig. 3) and start separating from each other at somewhere between $x=7$ and $x=7.5$ (see in Fig. 4). Let's consider familiar numbers $e$ in between 2.5 and 3 and $e^{2}$ in between 7 and 7.5. Due to this coincidence of $y=\log x$ and $\mathrm{f}(x)=0.19 x-0.01 x^{2}$ between $x=e$ and $x=$ $e^{2}$, the following can be stated. The logarithm of $x$ to base 10, approximately, equals $0.19 x-0.01 x^{2}$ where $x$ lies in between $e$ and $e^{2}$.

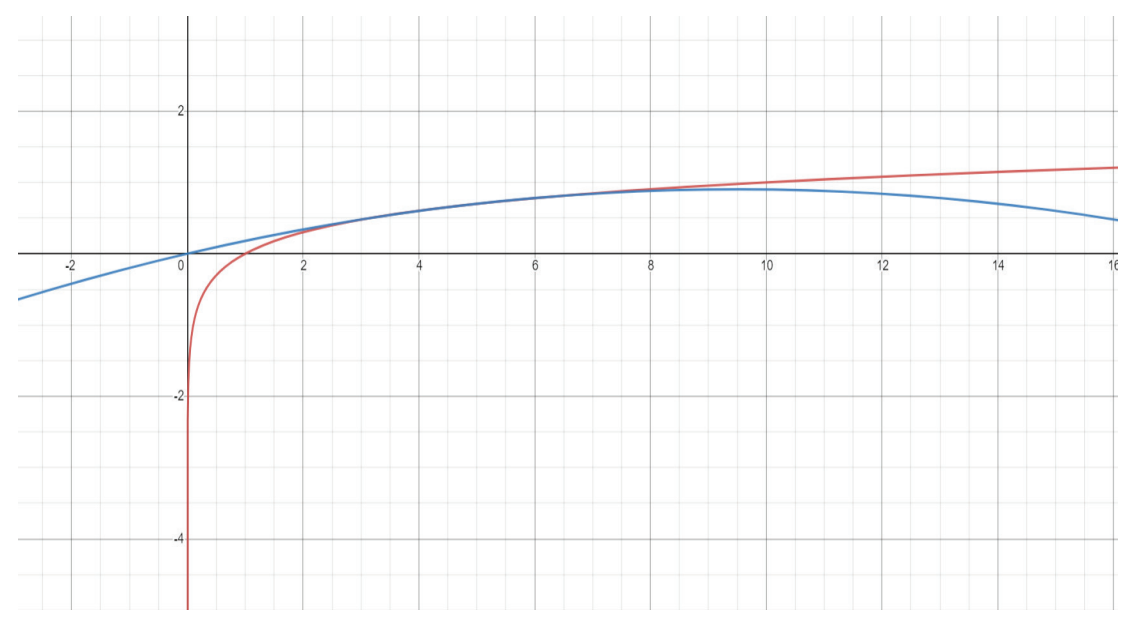

Figure 2: The parabolic graph $\mathrm{f}(\mathrm{x})=0.19 \mathrm{x}-0.01 \mathrm{x}^{2}$ (in blue) passing close to the common logarithmic function (in red).

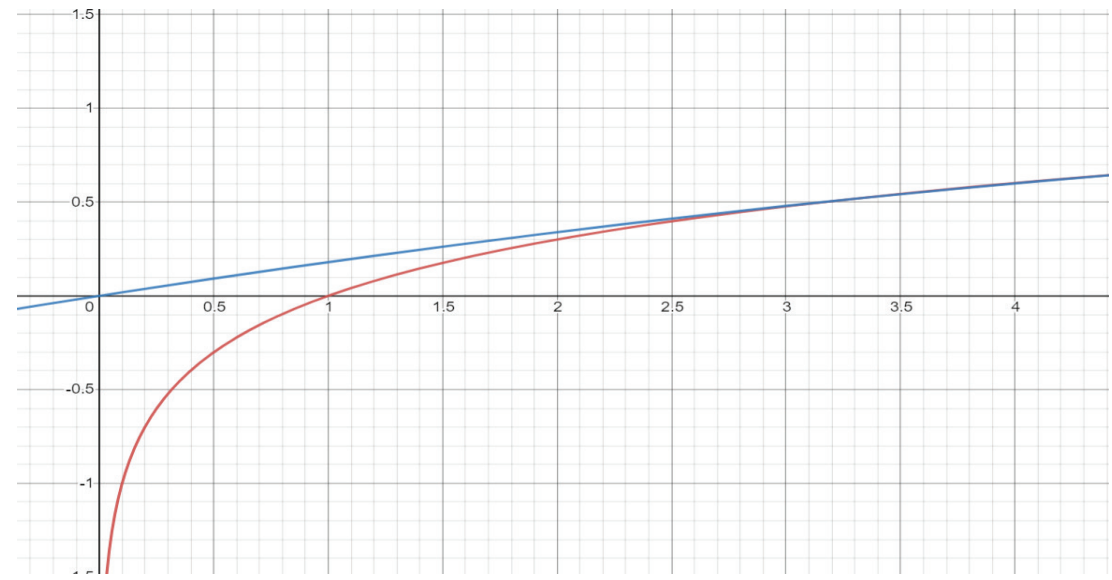

Figure 3: $f(x)=0.19 x-0.01 x^{2}$ and $y=\log x$ getting close to each other 


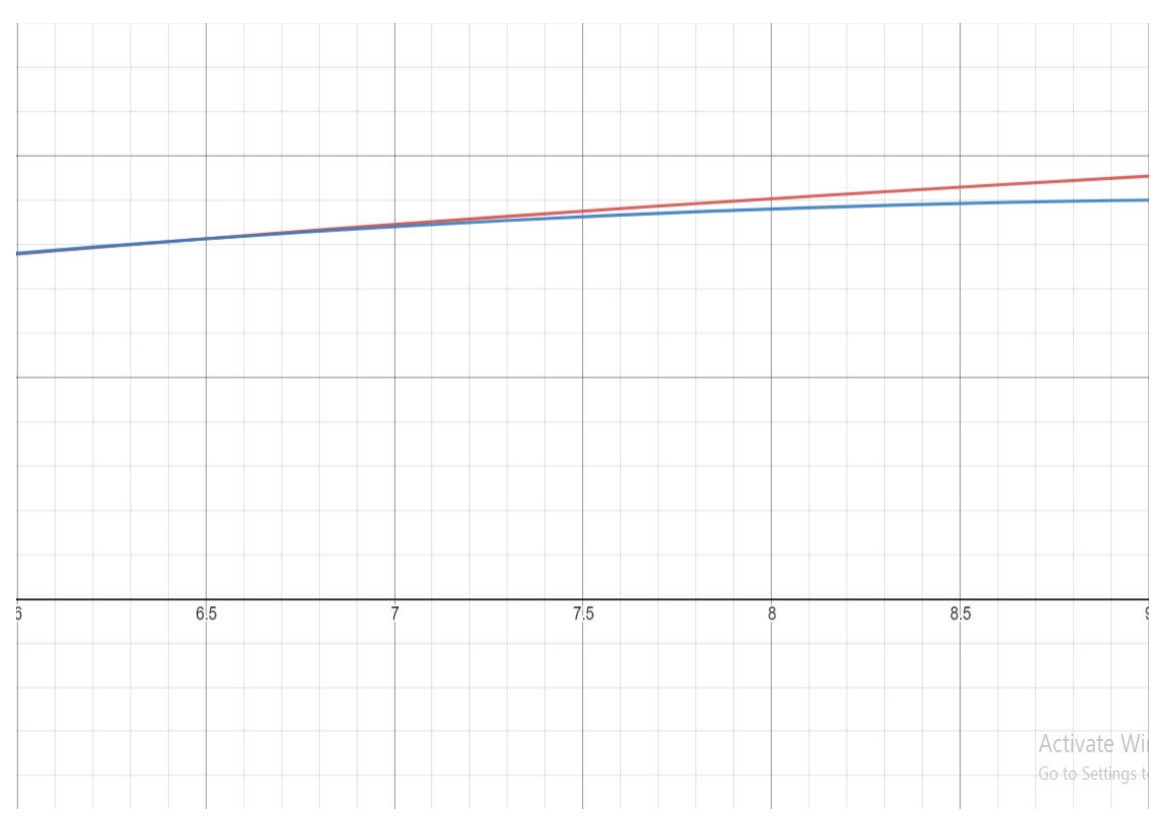

Figure 4: $f(x)=0.19 x-0.01 x^{2}$ and $y=\log x$ leaving each other

\section{Methodology}

The coincidence of $y=\log x$ with $\mathrm{f}(x)=0.19 x-0.01 x^{2}$ gives us a formula that predicts its values for $e<x<e^{2}$. Mathematically,

$$
\log x \approx \frac{(19-x) x}{100}, \text { for } e<x<e^{2}
$$

We just showed it graphically. But where did the equation come from in the first place? Let's take a detour and talk about its generation. Take approximate values of $\log$ base 10 for whole number $x$ between $e$ and $e^{2}$.

$$
0.48,0.60,0.70,0.78,0.84
$$

These are the approximate values for $\log 3, \log 4, \log 5, \log 6$, and $\log 7$ respectively. Now, we multiply them by 100 to make things easy.

$$
48,60,70,78,84
$$

We will try to decode its pattern if it has one. The first differences of the given sequence are given below.

$$
12,10,8,6
$$


They clearly have their second difference constant, i.e. -2 . This means that it's a quadratic sequence, whose formula can be written as ' $a x^{2}+b x+c$ '. [8]

We know that,

$$
\begin{aligned}
& 2 a=-2 \\
& \therefore a=-1
\end{aligned}
$$

To find the values of rest of the constants, we will put the values of $x$ in the equation and compare them.

$$
\begin{aligned}
& 48=-1 \times 3^{2}+3 b+c \ldots . .(i) \\
& 60=-1 \times 4^{2}+4 b+c \ldots \ldots(i i)
\end{aligned}
$$

After solving both equations, we get

$$
b=19 \text { and } c=0 .
$$

Substituting the values of constants, the formula becomes $\mathrm{f}(x)=19 x-x^{2}$, and since we had multiplied the sequence by 100 , we will now divide the formula by 100 , which leaves us with the required equation $\mathrm{f}(x)=19 x-x^{2} / 100$ for the values of $x$ starting from 3 through 7 . Now that we have already shown the graphical resemblance of $\log (x)$ and $\mathrm{f}(x)$, we can write the following:

$$
\log x \approx \frac{(19-x) x}{100}, \text { for } e<x<e^{2} \ldots(i)
$$

Now, you may think that this formula does not work for the real numbers less than $e$ and more than $e^{2}$, but that is not the case. Following algorithms that predict logarithm of real numbers less than $e$ and more than are based on the very formula.

Case I: When the number is less than $e$ and more than 0 (say $a$ ), multiply it by $k$ such that $e<k<e^{2}$ and $e<a \times k<e^{2}$. Then, the difference of logarithms of $a \times k$ and $k$ will give the logarithm of $a$ to base 10 .

$$
\begin{aligned}
& \log a=\log \frac{a \times k}{k}=\log a \times k-\log k[5,7] \\
& \therefore \log a \approx \frac{(19-a \times k) a \times k}{100}-\frac{(19-k) k}{100} \ldots . \text { (ii) }
\end{aligned}
$$

Case II: When the number is more than $e^{2}$ and less than 10 (say $b$ ), multiply it by $k$ such that $e<k<e^{2}$ and $e<\frac{b \times k}{10}<e^{2}$. Then, the difference of logarithms of $b \times k$ and $k$ will give the logarithm of $b$ to base 10 .

$$
\log b=\log \frac{b \times k}{k}=\log b \times k-\log k=1+\log \frac{b \times k}{10}-\log k
$$




$$
\therefore \log b \approx 1+\frac{\left(19-\frac{b \times k}{10}\right) \frac{b \times k}{10}}{100}-\frac{(19-k) k}{100} \text {....(iii) }
$$

Case III: When the number is more than 10 (say $c$ ), divide it by the suitable multiple of ten $\left(10^{\mathrm{N}}\right.$ : $\mathrm{N}$ is a Natural number) such that $0<\frac{c}{10^{N}}<10$. If $\frac{c}{10^{N}}$ is the number less than $e$ and more than 0 ,

$$
\log c \approx N+\frac{\left(19-\frac{c}{10^{N}} \times k\right) \frac{c}{10^{N}} \times k}{100}-\frac{(19-k) k}{100}
$$

If $\frac{c}{10^{N}}$ is more than $e$ and less than $e^{2}$,

$$
\log c \approx N+\frac{\left(19-\frac{c}{10^{N}}\right) \frac{c}{10^{N}}}{100} .
$$

And, if $\frac{c}{10^{N}}$ is more than $e^{2}$ and less than 10 ,

$$
\log c \approx N+1+\frac{\left(19-\frac{c}{10^{N}} \times k\right) \frac{c}{10} \times k}{10}-\frac{(19-k) k}{100} \ldots . \text { (iv) }
$$

Case IV: For bases other than 10 ( say $n$ ), the ratio of common logarithm of the given number to that of the base will give the logarithm of the given number to base $n$.

$$
\log _{n} x=\frac{\log x}{\log n} \ldots . .(\mathrm{v}) \quad[5,6,7]
$$

The examples below are given one from above each case:

A) Using equation number (i),

$$
\left.\log 4.15 \approx(19-4.15) \times \frac{4.15}{100}=0.616275 \text {. ( with } 0.28 \% \text { error }\right)
$$

B) Using equation number (ii),

$$
\begin{aligned}
\log 1.95 & =\log (1.95 \times 3)-\log 3=\log 5.85-\log 3 \\
\approx & \left.(19-5.85) \times \frac{5.85}{100}-(19-3) \times \frac{3}{100}=0.289275 . \text { (with } 0.26 \% \text { error }\right)
\end{aligned}
$$


C) Using equation number (iii),

$$
\begin{aligned}
\log 8.1 \times & 4-\log 4=1+\log 3.24-\log 4 \approx 1+(19-3.24) \times \frac{3.24}{100}-(19-4) \times \frac{4}{100} \\
& =0.910624 . \text { ( with } 0.23 \% \text { error) }
\end{aligned}
$$

D) Using equation number (iv),

$$
\begin{aligned}
\log 2345 & =3+\log 2.345=3+\log 2.345 \times 2.9-\log 2.9=3+\log 6.8005-\log 2.9 \\
\approx 3 & +(19-6.8) \times \frac{6.8}{100}-(19-2.9) \times \frac{2.9}{100}=3.3627 .(\text { with } 0.22 \% \text { error })
\end{aligned}
$$

E) Using equation number (v),

$$
\begin{aligned}
\log _{8.2} 4567 & =\frac{\log 4567}{\log 8.2} \approx \frac{3+(19-4.567) \times \frac{4.567}{100}}{\log 8.2 \times 4-\log 4}=\frac{3.659155}{1+\log 3.28-\log 4} \\
& =\frac{3.659155}{1+(19-3.28) \times \frac{3.28}{100}-0.60}=\frac{3.659155}{0.915616} \\
& =3.99638 . .(\text { with } 0.20 \% \text { error })
\end{aligned}
$$

The following formula was used to calculate the error:

$$
\text { Error }=\frac{\mid \text { Actual value }- \text { predicted value } \mid}{\text { Actual value }} \times 100 \%
$$

Finding suitable $k$ for case I and case II can be tricky sometimes, but hit and trial method can give us suitable $k$ easily. Taking the range of $x$ from $e$ to $e^{2}$ in consideration, this formula generates maximum $1.9 \%$ error.

\section{Conclusion}

The overlapping of graph of $\mathrm{f}(x)=0.19 x-0.01 x^{2}$ with that of $y=\log x$ gives us a formula and algorithms that use basic mathematical operations. Now, we can calculate the logarithm of real numbers to any real number bases with minimum possible error. People find remembering the log values difficult especially, in different examinations where calculators are not allowed. But, now, just a formula with some easy algorithms, and the difficulties related to remembering the values of logarithmic function for different numbers to different bases are solved. 


\section{References}

1. "The Ultimate Guide to Logarithm-Theory \& Applications", Math Vault, 8 May 2016, retrieved 24 July 2019

2. Shailesh S. (2002), A Primer on Logarithms, Hyderabad: Universities Press

3. Hall A.G., Frink F.G. (1909). "Chapter IV. Logarithms [23] Common logarithms". Trigonometry. Part I: Plane Trigonometry. New York: Henry Holt and Company. p. 31.

4. Hardy G.H. and Wright E.M (1975) An Introduction to the Theory of Numbers, 4th Ed., Oxford, footnote to paragraph 1.7: "log x is, of course, the 'Naperian' logarithm of $x$, to base e. 'Common' logarithms have no mathematical interest".

5. Bajracharya D.R., Shreshtha R.M., Singh M.B., Sthapit Y.R. and Bajracharya B.C. (2020) Basic Mathematics, Grade XI, p. 82 and p.83, Sukunda Publication and Distributor Pvt. Ltd..

6. Kafle J, Adhikary N, Kandel HP, Chapagai S, Karna R (2015) Principles of Business Mathematics, Kriti Prakashan, Bagbazar, Kathmandu.

7. Singh M. (1999) Pioneer Mathematics for class XI and XII, Dhanpat Rai and Company, India.

8. Siyavula Textbooks: Grade 11 Maths. P. 14-15 